Integr. equ. oper. theory 34 (1999) 165-186

$0378-620 \times / 99 / 020165-22 \$ 1.50+0.20 / 0$

(C) Birkhäuser Verlag, Basel, 1999

\title{
THE WEYL GROUP AND THE NORMALIZER OF A CONDITIONAL EXPECTATION
}

\author{
M. ARGERAMI AND D. STOJANOFF
}

\begin{abstract}
We define a discrete group $W(E)$ associated to a faithful normal conditional expectation $E: M \rightarrow N$ for $N \subseteq M$ von Neuman algebras. This group shows the relation between the unitary group $\mathcal{U}_{N}$ and the normalizer $\mathcal{N}_{E}$ of $E$, which can be also considered as the isotropy of the action of the unitary group $\mathcal{U}_{M}$ of $M$ on $E$. It is shown that $W(E)$ is finite if $\operatorname{dim} \mathcal{Z}(N)<\infty$ and bounded by the index in the factor case. Also sharp bounds of the order of $W(E)$ are founded. $W(E)$ appears as the fibre of a covering space defined on the orbit of $E$ by the natural action of the unitary group of $M . W(E)$ is computed in some basic examples.
\end{abstract}

\section{Introduction}

Let $N \subseteq M$ be von Neumann algebras and $E: M \rightarrow N$ a conditional expectation. Denote by $\mathcal{U}_{M}$ the unitary group of $M$ and by $\mathcal{N}_{E}$ the group

$$
\mathcal{N}_{E}=\left\{u \in \mathcal{U}_{M}: E\left(u x u^{*}\right)=u E(x) u^{*}, x \in M\right\},
$$

called the normalizer of $E$. The group $\mathcal{N}_{E}$ has been already studied, between other authors, by A. Connes ([6]) and Kosaki ([14]) in relation with crossed product inclusions of algebras.

In [2], [1] and [15] some differential geometric problems were related with the index theory of conditional expectations. The paper [15] is devoted to the study of the geometry of the orbit of each $E \in E(M)$ under the action of $\mathcal{U}_{M}$ given by

$$
L_{u} E=A d_{u} \circ E \circ A d_{u}^{-1}, \quad \text { for } \quad u \in \mathcal{U}_{M}
$$

where $A d_{u}(m)=u m u^{*}$, for $u \in \mathcal{U}_{N}$ and $m \in M$. These orbits are models for regular homogeneous reductive spaces. Note that the isotropy group for the action $L$ at $E$ is the group $\left\{u \in \mathcal{U}_{M}: L_{u}(E)=E\right\}$, which is exactly the normalizer $\mathcal{N}_{E}$. We consider the Jones' projection $e$ associated with $E$. It is a selfadjoint projection in the algebra $M_{1}$, the extension of $M$ by $N$ and $E$. The orbit

$$
\mathcal{U}_{M}(e)=\left\{u e u^{*}: u \in \mathcal{U}_{M}\right\}
$$


is a fibre bundle over the orbit of $E, \mathcal{O}_{E}=\left\{L_{u}(E): u \in \mathcal{U}_{M}\right\}$, via the formula

$$
\left(u e u^{*}\right) m\left(u e u^{*}\right)=L_{u} E(m)\left(u e u^{*}\right) \quad \text { for } \quad m \in M \text { and } u \in \mathcal{U}_{N} \text {, }
$$

which gives rise to the map $F: \mathcal{U}_{M}(e) \rightarrow \mathcal{O}_{E}$ given by $F\left(u e u^{*}\right)=L_{u}(E)$. Note that (see [2]) $\mathcal{U}_{M}(e)$ is a submanifold of the full orbit of $e$ in $M_{1}$, if and only if the index of $E$ is finite (see Definition 4.1 below).

In case that also $N^{\prime} \cap M \subseteq N$, the map $F$ defines a covering space ([2]). Therefore the fibre of $E$ is a discrete space denoted by

$$
\mathcal{P}(E)=\left\{u e u^{*}: u \in \mathcal{N}_{E}\right\} \subseteq \mathcal{U}_{M}(e)
$$

In this paper we remove the hypothesis $N^{\prime} \cap M \subseteq N$. We characterize the connected components of the group $\mathcal{N}_{E}$ and of $\mathcal{P}(E)$, which is a also a group, in terms of the unitary elements of the centralizer of $E$ (see [5] or [9]):

$$
M_{E}=\left\{x \in N^{\prime} \cap M: E(x m)=E(m x) \text { for all } m \in M\right\} \text {. }
$$

Indeed, the connected component of 1 in $\mathcal{N}_{E}$ is the group

$$
\mathcal{H}_{E}=\mathcal{U}_{N} \cdot \mathcal{U}_{M_{E}}=\left\{v w: v \in \mathcal{U}_{N} \text { and } w \in \mathcal{U}_{M_{E}}\right\}
$$

and the connected component of $\epsilon$ in $\mathcal{P}(E)$ is the suborbit of $\mathcal{U}_{M}(e)$ defined by the action of $\mathcal{H}_{E}$ :

$$
\mathcal{U}_{\mathcal{H}_{E}}(e)=\left\{u e u^{*}: u \in \mathcal{H}_{E}\right\}
$$

(see Prop. 2.6 and Thm. 4.7).

Also, $\mathcal{U}_{\mathcal{H}_{E}}(e)$ is a normal subgroup of $\mathcal{P}(E)$. We consider the quotient group

$$
W(E)=\mathcal{P}(E) / \mathcal{U}_{\mathcal{H}_{E}}(e) \simeq \mathcal{N}_{E} / \mathcal{H}_{E}
$$

This group, which we call the Weyl group of $E$, seems to give good information about the relation between $\mathcal{U}_{N}$ and $\mathcal{N}_{E}$, which is necessary in order to study the geometry of the orbit $\mathcal{O}_{E}$. For example, reductive structures can be defined on $\mathcal{O}_{E}$ when $M$ is finite. Also a covering space over $\mathcal{O}_{E}$ with fibres $W(E)$ can be constructed (without the hypotesis $\left.N^{\prime} \cap M \subseteq N\right)$.

In most interesting examples, the information obtained from $W(E)$ about the inclusion $N \subseteq M$ is poor. For example $W(E)$ is trivial if $N$ and $M$ are $\mathrm{II}_{1}$ factors and Ind $E<4$ (see Example 6.6). Nevertheless, in the finite index case, the iterated basic construction (see $[18,1.2 .2]$ ) produces a tower of algebras

$$
N \subseteq M=M_{0} \subseteq M_{1} \subseteq M_{2} \subseteq \ldots \subseteq M_{n} \subseteq \ldots
$$

with finite index conditional expectations $E_{n} \in E\left(M_{n}, M_{n-1}\right)$, for $n \geq 1$. Considering $F_{n} \in E\left(M_{n}, N\right)$ given by $F_{n}=E \circ E_{1} \circ \ldots \circ E_{n}$, one obtains a tower of finite Weyl groups (see 2.9 and 2.10 )

$$
W(E)=W\left(F_{0}\right) \subseteq W\left(F_{1}\right) \subseteq W\left(F_{2}\right) \subseteq \ldots \subseteq W\left(F_{n}\right) \subseteq \ldots
$$


which seems to be a rich invariant for the original inclusion $N \subseteq M$ and the expectation $E$. Note that the inclusions $N \subseteq M_{n}$ don't verify in general that $N^{\prime} \cap M_{n} \subseteq N$. Then, in order to study the tower of groups one needs to know the properties of the Weyl group for inclusions $N \subseteq M$ which don't verify that $N^{\prime} \cap M \subseteq N$, which is the case considered in this work.

The applications of the results of this work in order to study the tower of Weyl groups and the geometry and topology of the orbits $\mathcal{O}_{E}$ will appear in a forthcoming paper.

The Weyl group $W(E)$ will be considered with the discrete topology, since this is the topology that makes the isomorphism of (1) bicontinuous (see Prop. 2.6). When $M$ is the crossed product of $N$ by a properly outer discrete group of automorphisms, $W(E)$ contains the original group. The two groups are isomorphic when $G$ is finite and $N$ is a. factor.

If Ind $E<\infty$ and $\operatorname{dim}(\mathcal{Z}(N))<\infty$ then $W(E)$ is finite (Thm. 5.3). Moreover, if $N$ is a factor we show (Thm. 5.4) that, if $|W(E)|$ denotes the order of $W(E)$,

$$
|W(E)| \leq \inf \{\lambda: \lambda \in \sigma(\text { Ind } E)\}=\left\|\operatorname{Ind} E^{-1}\right\|^{-1},
$$

where Ind $E$ is the operator valued index of $E$ (see [4] or Definition 4.1 below). We also get sharp bounds for $|W(E)|$ in terms of Ind $E$ and $\operatorname{dim}(\mathcal{Z}(N))$ (see Thm. 5.7).

The Weyl group and the normalizer of $E$ are computed in several examples of conditional expectations $E$. We mention briefly some of them:

1. (see 6.1) Let $\mathcal{R}$ be a factor, and consider the inclusion

$$
N=\mathcal{R}^{n} \subseteq \mathcal{R}^{n \times n}=M
$$

where the conditional expectation $E$ acts by "compression to the diagonal". Then $W(E)$ is the group $\mathcal{S}_{n}$ of permutations of $n$ elements.

2. (see 6.4) Let $N$ be a factor and consider now the inclusion

$$
N \subseteq M=N^{n \times n}
$$

with the conditional expectation being the "normalized operator valued trace". Then $W(E)$ consists of a single element and the normalizer of $E$ can be described as

$$
\mathcal{N}_{E}=\left\{\left(n, a_{i j}\right)_{i j}: n \in \mathcal{U}_{N} \text { and }\left(a_{i j}\right) \in \mathbb{C}^{n \times n} \text { is unitary }\right\}
$$

3. (see 6.5) Take a von Neumann algebra $N$ and a discrete group $G$ of outer (and free) automorphisms of $N$, and consider $M=N \times_{\alpha} G$, with the canonical conditional expectation. Then $G \subseteq W(E)$. In general $W(E)$ is bigger than $G$, also when $G$ is finite. Nevertheless, when $G$ is finite and $N$ is a factor, one has that $G \simeq W(E)$.

4. (see 6.6) Let $N \subseteq M$ be $\mathrm{II}_{1}$ factors and $E \in E(M, N)$ with Ind $E<4$. Then

(a) If the principal graph is neither of type $\mathrm{A}_{3}$ nor $\mathrm{D}_{4}$, then $W(E)$ is trivial. 
(b) If Ind $E=2$ and the principal graph is of type $A_{3}$, then $W(E)=\mathbb{Z}_{2}$.

(c) If Ind $E=3$ and the principal graph is of type $\mathrm{D}_{4}$, then $W(E)=\mathbb{Z}_{3}$.

In Section 2 we define the Weyl group in terms of its action on $N$ by automorphisms and we show its basic properties.

In Section 3 we show the properties of the group $\mathcal{P}(E)$ and its relation with $W(E)$ and the basic construction for $E$.

In Section 4 we consider the finite index case, particularly in order to get information about the inclusion $\mathcal{P}(E) \subseteq N^{\prime} \cap M_{1}$. This study is useful for computing $W(E)$ in the finite index case.

In Section 5 we get conditions for finiteness of $W(E)$ and bounds for its order.

Section 6 is fully dedicated to present examples. In particular there is an example where Ind $E<\infty$ but $W(E)$ is infinite, and also an example where the bound for the order of $W(E)$ obtained in Section 5 is attained.

\section{The group $W(E)$, basic properties and examples.}

Let $M$ be a von Neumann algebra. Denote by $\mathcal{U}_{M}$ the unitary group of $M, G_{M}$ the group of its invertible elements and $\mathcal{Z}(\mathcal{M})$ the center of $M$. Denote by $E(M)$ the set of faithful normal conditional expectations defined on $M$ onto its subalgebras. Consider the action $L: \mathcal{U}_{M} \times E(M) \rightarrow E(M)$, given by

$$
L_{u} E=A d_{u} \circ E \circ A d_{u^{*}}, \quad \text { for } E \in E(M) \text { and } u \in \mathcal{U}_{M} \text {, }
$$

where $A d_{u}$ denotes the inner automorphism of $M$ induced by $u$. For any $E \in E(M)$, denote by $\mathcal{O}_{E}=\left\{L_{u} E: u \in \mathcal{U}_{M}\right\}$ the orbit of $E$ by this action. In order to study the geometrical properties of the orbits $\mathcal{O}_{E}$, we consider, for each $E \in E(M)$, the map

$$
\pi_{E}: \mathcal{U}_{M} \rightarrow E(M), \quad \text { given by } \quad \pi_{E}(u)=L_{u} E, \quad u \in \mathcal{U}_{M}
$$

We are interested in characterizing the isotropy of this action. Let $N \subseteq M$ be a von Neumann subalgebra of $M$, and $E(M, N)$ the set of faithful normal conditional expectations $E: M \rightarrow N$. Denote $N^{\mathrm{c}}=N^{\prime} \cap M$.

Definition 2.1 Let $N \subseteq M$ be von Neumann algebras and $E \in E(M, N)$. Denote by $\mathcal{N}_{E}$ the group

$$
\begin{aligned}
\mathcal{N}_{E} & =\left\{u \in \mathcal{U}_{M}: \pi_{E}(u)=E\right\} \\
& =\left\{u \in \mathcal{U}_{M}: E\left(u x u^{*}\right)=u E(x) u^{*}, x \in M\right\},
\end{aligned}
$$

called the normalizer of $E$.

The group $\mathcal{N}_{E}$ has been already studied, between other authors, by $\mathrm{A}$. Connes ([6]) and Kosaki ([14]) in relation with crossed product inclusions of algebras. 
It is clear that $\mathcal{U}_{N}$ is contained in $\mathcal{N}_{E}$. In some cases (see example 6.6) these two groups coincide. On the other hand, if $u \in \mathcal{N}_{E}$, then $u N u^{*}=N$ (the converse of this property is true only when $\left.N^{\prime} \cap M \subseteq M([6,1.5 .6])\right)$. Indeed, this can be easily seen using that for all $v \in \mathcal{U}_{M}$, the image of $L_{v}(E)$ is $v N v^{*}$.

In order to characterize $\mathcal{N}_{E}$, we consider the map

$$
\left.A d\right|_{N}: \mathcal{N}_{E} \rightarrow \operatorname{Aut}(N) \text {. }
$$

Here, for each $u \in \mathcal{N}_{E}$, we denote by $A d_{u}$ the inner automorphisms defined by $u$ on $M$, but restricted to $N$. That is, $A d_{u}(n)=u n u^{*}$, for $n \in N$ and $u \in \mathcal{N}_{E}$. In order to study the relationship between $\mathcal{U}_{N}$ and $\mathcal{N}_{E}$, we consider the composition of $\left.A d\right|_{N}$ with the canonical projection from $A u t(N)$ onto the quotient group $\operatorname{Out}(N)=\operatorname{Aut}(N) / \operatorname{Inn}(N)$, where $\operatorname{Inn}(N)$ is the group of inner automorphisms of $N$ :

Definition 2.2 Let $N \subseteq M$ be von Neumann algebras and $E \in E(M, N)$. Denote by $\rho_{E}: \mathcal{N}_{E} \rightarrow \operatorname{Out}(N)$ the group homomorphism given by

$$
\rho_{E}(u)=\left[A d_{u}\right]=A d_{u} \cdot \operatorname{Inn}(N), \quad \text { for } u \in \mathcal{N}_{E} .
$$

We can now define a Weyl group of $E$ :

Definition 2.3 Let $N \subseteq M$ be von Neumann algebras and $E \in E(M, N)$. We denote by $W(E)$ the group $\rho_{E}\left(\mathcal{N}_{E}\right)$ and call it the Weyl group of the expectation $E$. We consider on $W(E)$ the discrete topology.

Remark 2.4 It is well known (see [12, 10.5.73]) that if we consider on $\operatorname{Aut}(N)$ the norm topology (as linear operators on $N$ ), then the quotient topology induced on $O u t(N)$ is discrete. Therefore the map $\rho_{E}$ of 2.2 is continuous when the norm topology is considered on $\mathcal{N}_{E}$.

Definition 2.5 Denote by

$$
M_{E}=\left\{x \in N^{\prime} \cap M: E(x m)=E(m x) \text { for all } m \in M\right\},
$$

named the "centralizer" of $E$ in the literature ([5] or [9]). $M_{E}$ is a von Neumann algebra since $E$ is normal. Denote by $\mathcal{H}_{E}$ the group

$$
\mathcal{H}_{E}=\mathcal{U}_{N} \cdot \mathcal{U}_{M_{E}}=\left\{v w: v \in \mathcal{U}_{N} \text { and } w \in \mathcal{U}_{M_{E}}\right\}
$$

It is easy to see that $\mathcal{H}_{E}$ is connected, since $\mathcal{U}_{M_{E}}$ is the unitary group of the von Neumann algebra $M_{E}$. Also $\mathcal{H}_{E}$ is a closed (in norm) subgroup of $\mathcal{N}_{E}$.

Proposition 2.6 Let $N \subseteq M$ be von Neumann algebras, $E \in E(M, N)$ and $\rho_{E}$ as in 2.2. Then $\operatorname{ker}\left(\rho_{E}\right)=\mathcal{H}_{E}$ and $\rho_{E}$ induces the isomorphism

$$
\Phi: \mathcal{N}_{E} / \mathcal{H}_{E} \rightarrow W(E)
$$

which is an homeomorphism when considered $\mathcal{N}_{E} / \mathcal{H}_{E}$ with the quotient topology of the norm topology of $\mathcal{N}_{E}$.

Moreover, the connected component of $\mathcal{N}_{E}$ at any $u \in \mathcal{N}_{E}$ is exactly $u . \mathcal{H}_{E}$. The distance between different connected components is greater than 1 . 
Proof. Let $u \in \operatorname{ker}\left(\rho_{E}\right)$. Then there exists $v \in \mathcal{U}_{N}$ such that $\left.A d_{u}\right|_{N}=A d_{v}$. Hence $u v^{*} \in$ $N^{\mathrm{c}} \cap \mathcal{N}_{E}$. It is easy to see that $N^{\mathrm{c}} \cap \mathcal{N}_{E}=\mathcal{U}_{M_{E}}$. Therefore $\operatorname{ker}\left(\rho_{E}\right)=\mathcal{H}_{E}$, since the other inclusion is clear.

This proves that $\Phi$ is a group isomorphism. Easy computations show that, if $u, v \in$ $\mathcal{N}_{E}$, then

$$
\left\|\left.A d_{u}\right|_{N}-\left.A d_{v}\right|_{N}\right\| \leq\left\|\delta_{u^{*}}\right\| \leq 2\left\|1-u^{*} v\right\|=2\|u-v\|,
$$

where $\delta_{u^{*} v}$ denotes the inner derivation of $M$ given by $\delta_{u^{*} v}(x)=x u^{*} v-u^{*} v x$, for $x \in M$. It is well known that if two automorphisms of $N$ lie at (norm) distance less than 2, then their images in $O u t(N)$ coincide. We can deduce that if $\rho_{E}(u) \neq \rho_{E}(v)$ then $\|u-v\| \geq 1$. Using that the group $\mathcal{H}_{E}$ is connected, we have that each set $u . \mathcal{H}_{E}$ is open, closed and connected, and the proof is complete

Remark 2.7 In order to characterize $\mathcal{N}_{E}$, it is enough to kwow $\mathcal{U}_{N}, \mathcal{U}_{M_{E}}$ and $W(E)$. On the other hand, the group $W(E)$ is an invariant for the conditional expectation $E$. Moreover, the characterization of the connected component of 1 in $\mathcal{N}_{E}$ as the group $\mathcal{H}_{E}$ is important in order to study the geometrical properties of the orbit $\mathcal{O}_{E}$. Particularly the existence of reductive structures (see [3]).

The following proposition shows that if we "sum inclusions" we get the sum of their Weyl groups. We will use this result later in order to get bounds for the order of the Weyl group.

Proposition 2.8 Let $N \subseteq M$ be von Neumann algebras and $E \in E(M, N)$. Suppose that $p_{1}, \ldots, p_{n}$ are orthogonal projections in $\mathcal{Z}(N) \cap \mathcal{Z}(M)$ such that $\sum_{i} p_{i}=1$. Let $E_{i}: p_{i} M \rightarrow$ $p_{i} N$ be given by $E_{i}=\left.E\right|_{p_{i} M}$. Then $E_{i} \in E\left(p_{i} M, p_{i} N\right)$ for all $1 \leq i \leq n$ and

$$
W(E) \simeq \oplus_{i=1}^{n} W\left(E_{i}\right)
$$

Proof. The basic idea of the proof is the following: since for $1 \leq i \leq n, p_{i} \in \mathcal{Z}(N) \cap \mathcal{Z}(M)$, all the elements of $M$ are "diagonal" with respect to the projections $p_{i}$. Then we shall see that

$$
\mathcal{N}_{E} \simeq \oplus_{i=1}^{n} \mathcal{N}_{E_{i}} \quad \text { and } \quad \mathcal{H}_{E} \simeq \oplus_{i=1}^{n} \mathcal{H}_{E_{i}}
$$

where the isomorphism is just taking the diagonal entries of the unitaries considered in both cases. Using these facts, our statement follows by elementary theory of groups.

Easy calculations show that for all $1 \leq i \leq n$,

$$
E_{i} \in E\left(p_{i} M, p_{i} N\right) \quad, \quad \mathcal{N}_{E_{i}}=p_{i} \mathcal{N}_{E} \quad \text { and } \quad \mathcal{H}_{E_{i}}=p_{i} \mathcal{H}_{E}
$$

These facts need several verifications to be proved $\left(p_{i}\left(N^{\prime} \cap M\right)=\left(p_{i} N\right)^{\prime} \cap p_{i} M, p_{i} M_{E}=\right.$ $\left(p_{i} M\right)_{E_{i}}$, etc). But all are apparent using that all $p_{i} \in \mathcal{Z}(N) \cap \mathcal{Z}(M)$ and then $E(m)=$ $\sum_{i} E_{i}\left(p_{i} m\right)$, for all $m \in M$. This shows that the map

$$
\mathcal{N}_{E} \ni u \mapsto \oplus_{i} p_{i} u \in \oplus_{i=1}^{n} \mathcal{N}_{E_{i}}
$$


is well defined and maps $\mathcal{H}_{E}$ into $\oplus_{i=1}^{n} \mathcal{H}_{E_{i}}$. Injectivity and surjectivity in both cases is also easy to see. For example, if $u_{1} \oplus \ldots \oplus u_{n} \in \oplus_{i} \mathcal{N}_{E_{i}}$, then $u=\sum_{i} u_{i} \in \mathcal{U}_{M}$ and, for $m \in M$,

$$
E\left(u m u^{*}\right)=\sum_{i} E_{i}\left(p_{i} u m u^{*}\right)=\sum_{i} E_{i}\left(u_{i} p_{i} m u_{i}^{*}\right)=\sum_{i} u_{i} E_{i}\left(p_{i} m\right) u_{i}^{*}=u E(m) u^{*},
$$

and $u \in \mathcal{N}_{E}$, proving surjectivity. Surjectivity al the level of $\mathcal{H}_{E}$ is easier and injectivity is trivial.

Proposition 2.9 Let $N \subseteq L \subseteq M$ be von Neumann algebras, $E_{0} \in E(M, L), E \in E(L, N)$ and $F=E \circ E_{0} \in E(M, N)$. Then

$$
\mathcal{N}_{E}=\mathcal{N}_{F} \cap L \quad \text { and } \quad \mathcal{H}_{E}=\mathcal{H}_{F} \cap L
$$

and therefore

$$
W(E) \subseteq W(F)
$$

where the inclusion means that $W(E)$ is naturally isomorphic to a subgroup of $W(F)$.

Proof. It is clear using that $\left.F\right|_{L}=E$, that $\mathcal{N}_{F} \cap L \subseteq \mathcal{N}_{E}$ and $\mathcal{H}_{F} \cap L \subseteq \mathcal{H}_{E}$. On the other hand, let $u \in \mathcal{N}_{E}$, and $m \in M$. Then $u \in L$ and

$$
F\left(u m u^{*}\right)=E\left(u E_{0}(m) u^{*}\right)=u E\left(E_{0}(m)\right) u^{*}=u F(m) u^{*},
$$

hence $u \in \mathcal{N}_{F}$. Similarly one shows that $L_{E}=M_{F} \cap L$. Therefore $\mathcal{H}_{E}=\mathcal{H}_{F} \cap L=\mathcal{H}_{F} \cap \mathcal{N}_{E}$. Finally, the inclusion $\mathcal{N}_{E} \rightarrow \mathcal{N}_{F}$ induces the natural isomorphism

$$
W(E) \simeq \mathcal{N}_{E} / \mathcal{H}_{E}=\mathcal{N}_{E} /\left(\mathcal{H}_{F} \cap \mathcal{N}_{E}\right) \rightarrow \mathcal{N}_{F} / \mathcal{H}_{F} \simeq W(F)
$$

Remark 2.10 Let $N \subseteq M$ be $\mathrm{II}_{1}$ factors and $E \in E(M, N)$ of finite index. In the most interesting examples the information one gets from $W(E)$ about this inclusion is poor. For example $W(E)$ is trivial if Ind $E<4$ (see Example 6.6). But the iterated basic construction produces a tower of factors

$$
N \subseteq M \subseteq M_{1} \subseteq M_{2} \subseteq \ldots \subseteq M_{n} \subseteq \ldots
$$

and a sequence of conditional expectations $E_{n} \in E\left(M_{n}, M_{n-1}\right)$, where we can redefine $M=$ $M_{0}, N=M_{-1}$ and $E=E_{0}$ to have a coherent notation. These expectations verify that Ind $_{B D H} E_{n}=$ Ind $E_{n}=$ Ind $E$ for all $n \in I N$ (see 4.1 for definitions).

Consider $F_{n} \in E\left(M_{n}, M_{-1}\right)$ given by $F_{n}=E_{0} \circ E_{1} \circ \ldots \circ E_{n}$. Then Ind $F_{n}=$ Ind $E^{n+1}<\infty$. Note that $F_{n+1}=F_{n} \circ E_{n+1}$. Therefore, using Proposition 2.9, we obtain a tower of Weyl groups

$$
W(E)=W\left(F_{0}\right) \subseteq W\left(F_{1}\right) \subseteq W\left(F_{2}\right) \subseteq \ldots \subseteq W\left(F_{n}\right) \subseteq \ldots
$$

which seems to be a reach invariant for the original inclusion $N \subseteq M$ and the expectation $E$. Note that the inclusions $N \subseteq M_{n}$ verify that $N^{\prime} \cap M_{n} \neq \mathbb{C}$. Then, in order to study the tower of groups one needs to know the properties of the Weyl group for non irreducible inclusions or, in general, inclusions $N \subseteq M$ which don't verify that $N^{\mathrm{c}} \subseteq N$. 
Remark 2.11 Let $N \subseteq M \subseteq B(H)$ be von Neumann algebras where $H$ is a separable Hilbert space and let $F \in E(M, N)$. Denote by

$$
L=\left\{N \cup \mathcal{N}_{F}\right\}^{\prime \prime} .
$$

Clearly $N \subseteq L \subseteq M$ and we can consider $E=\left.F\right|_{L} \in E(L, N)$. Then there exists $E_{0} \in$ $E(M, L)$ such that $F=E \circ E_{0}$ and using Proposition 2.9 one deduces that

$$
W(E) \simeq W(F)
$$

Indeed, let $\phi$ be a faithful normal state (or semifinite weight) on $N$ and $\psi=\phi \circ F$ the corresponding state on $M$. Then the modular group of $\psi$ leaves $\mathcal{N}_{E}$ and $N$ invariant, since it commutes with $F$. Then, by Takesaki theorem (see [19]), there exists a unique $E_{0} \in E(M, L)$ such that $\psi \circ E_{0}=\psi$. But then both $E \circ E_{0}$ and $F$ are $\psi$ invariant and must coincide by the unicity assertin of the above mentioned Takesaki theorem.

It would be reasonable to hope, since the full Weyl group is "contained" in $L$, that $W\left(E_{0}\right)$ were trivial. Nevertheless this is not true, as can be easily seen by regarding the fixed algebra of a factor $M$ by a finite outer group of automorphisms.

\section{The group $\mathcal{P}(E)$}

Let $N \subseteq M$ be von Neumann algebras and $E \in E(M, N)$. We assume the existence of a (fixed) faithful normal state $\psi$ on $N$, and consider the faithful normal state $\varphi=\psi \circ E$ on $M$.

Consider $M$ and $N$ with their GNS representation given by the state $\varphi$, acting on the Hilbert space $L^{2}(M, \varphi)$. Define the Jones' projection $e$ to be the ortogonal projection with range $L^{2}(N, \psi)$ considered as a subspace of $L^{2}(M, \varphi)$. We also consider the Basic Construction for $e$, the algebra $M_{1}=\{M, e\}^{\prime \prime}$, the von Neumann algebra generated by $M$ and $e$ in $L\left(L^{2}(M, \varphi)\right)$. This definition of $e$ and $M_{1}$ is equivalent to the common "bimodule" aproach (see [4] or [9]), but seems to be more simple for our purposes.

Remark 3.1 Let $N \subseteq M$ be von Neumann algebras and $E \in E(M, N)$. Then the Jones' projection $e$ associated with $E$ satisfies the usual properties (see, for example, [8, 3.6.1], [18, 1.1.3] or $[2,1.1])$ :

1. For $a \in M$, eae $=E(a) e$.

2. $N=\{e\}^{\prime} \cap M$.

3. If $a \in M$, $a e=0$ implies $a=0$.

4. $x \mapsto x e$ is a ${ }^{*}$-isomorphism from $N$ onto $e M_{1} e$.

5. The subalgebra $M_{0}=M+M e M$ is ultraweakly dense in $M_{1}$ 
Denote by $\mathcal{U}_{M}(e)=\left\{u e u^{*}: u \in \mathcal{U}_{M}\right\}$, the orbit of $e$ by the action of $\mathcal{U}_{M}$ by conjugation. It is proved in [2] that $\mathcal{U}_{M}(e)$ is a submanifold of the full unitary orbit of $e$ in $M_{1}$, if and only if the index of $E$ is finite (see Definition 4.1 below).

Consider the map $\pi_{e}: \mathcal{U}_{M} \rightarrow \mathcal{U}_{M}(e)$ given by $\pi_{e}(u)=u e u^{*}$, for $u \in \mathcal{U}_{M}$. The orbit $\mathcal{U}_{M}(e)$ is a fibre bundle over the orbit $\mathcal{O}_{E}$ of $E$ via the map

$$
F: \mathcal{U}_{M}(e) \rightarrow \mathcal{O}_{E}
$$

defined as follows: given $u \in \mathcal{U}_{M}$ and $p=u e u^{*} \in \mathcal{U}_{M}(e)$ we denote by $F(p)$ the unique element of $E(M)$ such that

$$
F(p)(m) p=p m p \quad \text { for all } \quad m \in M .
$$

Indeed, we have the following commutative diagram:

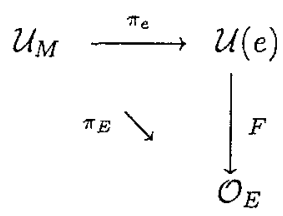

Definition 3.2 Let $N \subseteq M$ be von Neumann algebras and $E \in E(M, N)$. Denote by $\mathcal{P}(E)$ the set

$$
\mathcal{P}(E)=\left\{u e u^{*}: u \in \mathcal{N}_{E}\right\} .
$$

This set is the fibre of the fibration $F$ of diagram (2) over $E$.

Remark 3.3 Let $N \subseteq M$ be von Neumann algebras and $E \in E(M, N)$. Then

1. The group $\mathcal{U}_{N}$ is a normal subgroup of $\mathcal{N}_{E}$. Also the map $\left.\pi_{e}\right|_{\mathcal{N}_{E}}: \mathcal{N}_{E} \rightarrow \mathcal{P}(E)$, is onto and verifies that given $u, v \in \mathcal{N}_{E}, \pi_{e}(u)=\pi_{e}(v)$ iff $u v^{*} \in \mathcal{U}_{N}$. Then it induces on $\mathcal{P}(E)$ a group structure. Indeed the product is defined by

$$
\pi_{e}(u) \cdot \pi_{e}(v)=\pi_{e}(u v)
$$

for $u, v \in \mathcal{N}_{E}$. Note that as a group

$$
\mathcal{P}(E) \simeq \mathcal{N}_{E} / \mathcal{U}_{N}
$$

In case that $E$ has finite index (see 4.1) it is proved in [2] that the the map $\pi_{e}$ has local cross sections. So that this isomorphism is also an homeomorphism when considering $\mathcal{U}_{M}(e)$ with the norm topology of $M_{1}$ and $\mathcal{N}_{E} / \mathcal{U}_{N}$ with the topology induced by the norm topology of $\mathcal{N}_{E}$.

2. Since $e \in N^{\prime} \cap M_{1}$ and for $u \in \mathcal{N}_{E}, u N u^{*}=N$ and $u N^{\prime} u^{*}=N^{\prime}$, we can easily deduce that

$$
\mathcal{P}(E) \subseteq N^{\prime} \cap M_{1}
$$


3. If $N^{\mathrm{c}} \subseteq N$, then

$$
\mathcal{P}(E) \simeq W(E) .
$$

Indeed, since $M_{E} \subseteq N^{\mathbf{c}} \subseteq N$, we have $\mathcal{H}_{E}=\mathcal{U}_{N}$ and it must be $\mathcal{P}(E)=W(E)$ by 1 and Proposition 2.6.

4. Since $\mathcal{H}_{N}$ is a normal subgroup of $\mathcal{H}_{E}$, we have that

$$
W(E) \simeq \mathcal{N}_{E} / \mathcal{H}_{E} \simeq \frac{\mathcal{N}_{E} / \mathcal{U}_{N}}{\mathcal{H}_{E} / \mathcal{U}_{N}} \simeq \mathcal{P}(E) /\left(\mathcal{H}_{E} / \mathcal{U}_{N}\right)
$$

where $\mathcal{H}_{E} / \mathcal{U}_{N}$ is a subgroup of $\mathcal{P}(E)$. In other words, the Weyl group $W(E)$ is always a quotient of the fibre $\mathcal{P}(E)$. Note that $\mathcal{H}_{E} / \mathcal{U}_{N}$ can be identified with the orbit $\left\{u e u^{*}: u \in \mathcal{H}_{E}\right\}$. In the finite index case we shall see that this orbit is exactly the connected component of $e$ in $\mathcal{P}(E)$. Therefore the group $W(E)$ can be identified in this case with the set of connected components of the fibre $\mathcal{P}(E)$.

Let $p \in \mathcal{P}(E)$ and $u \in \mathcal{N}_{E}$ such that $p=u e u^{*}$. Then $p e=u E\left(u^{*}\right) e$. In order to study the properties of $p e$ we need the following Lemma:

Lemma 3.4 Let $N \subseteq M$ be von Neumann algebras and $E \in E(M, N)$ and $u \in \mathcal{N}_{E}$. Then

1. $u E\left(u^{*}\right) \in M_{E}$.

2. $u E\left(u^{*}\right)=E\left(u^{*}\right) u$.

3. $E(u) E\left(u^{*}\right) \in \mathcal{Z}(N)$.

4. If $N^{\mathrm{c}} \subseteq N$, then $u E\left(u^{*}\right)$ is a projection in $\mathcal{Z}(N)$ and

$$
u E\left(u^{*}\right)=E\left(u^{*}\right) u=E(u) E\left(u^{*}\right)=E\left(u^{*}\right) E(u)=u^{*} E(u)=E(u) u^{*}
$$

Proof. Let $b \in N$. Then

$$
u E\left(u^{*}\right) b e=u e u^{*} b e=u e\left(u^{*} b u\right) u^{*} e .
$$

Since $u^{*} N u=N$ and $e \in N^{\prime}$, we have

$$
u E\left(u^{*}\right) b e=u\left(u^{*} b u\right) e u^{*} e=b u E\left(u^{*}\right) e,
$$

So that by 3 of Remark $3.1, u E\left(u^{*}\right) \in N^{\mathrm{c}}$. To prove 2, note that

$$
u E\left(u^{*}\right)=u E\left(u^{*}\right) u^{*} u=E\left(u u^{*} u^{*}\right) u=E\left(u^{*}\right) u \text {. }
$$

3 ) is obvious since $E\left(N^{c}\right)=N^{\prime} \cap N=\mathcal{Z}(N)$. Let us prove 1. If $x \in M$, using 2 and 3, we have

$$
\begin{aligned}
E\left(u E\left(u^{*}\right) x\right) & =E\left(E\left(u^{*}\right) u x\right)=E\left(u^{*}\right) E(u x)=E\left(u^{*}\right) u E(x u) u^{*} \\
& =E(x u) E\left(u^{*}\right)=E\left(x u E\left(u^{*}\right)\right) .
\end{aligned}
$$

To see 4 , note that in the case $N^{\mathbf{c}} \subseteq N$ we have $u E\left(u^{*}\right) \in N$, so it has to be $E\left(u E\left(u^{*}\right)\right)=u E\left(u^{*}\right)$, and the assertion follows easily from the preceeding properties

Remark 3.5 Item 4 in the preceeding lemma is stated in Connes work [6, 1.5.5], where he also notes that if $N^{\mathrm{c}} \subseteq N$, then $u N u^{*}=N$ implies that $u \in \mathcal{N}_{E}$. 


\section{The finite index case}

Let $N \subseteq M$ be von Neumann algebras and $E: M \rightarrow N$ be a conditional expectation. $E$ is said to have finite index (and writen Ind $E<\infty$ ) if one of the following three equivalent conditions hold (see, for example, [18]):

1. There exists $0<K \in I R$ such that the linear map $K E-I d$ is positive on $M$.

2. There exists $0<L \in I R$ such that the linear map $L E-I d$ is completely positive on $M$.

3. There exists a $N$-quasi basis $\left\{m_{k}\right\}_{k \in K}$ for $M$ such that $\sum_{k \in K} m_{k}$ converges (see [4, $1.6],[20,1.2 .2]$ or $[18,1.1 .4]$ for the definition of $N$-quasi basis).

In this case $E$ is faithful and normal, that is $E \in E(M, N)$.

Definition 4.1 Let $N \subseteq M$ be von Neumann algebras and $E: M \rightarrow N$ be a conditional expectation such that Ind $E<\infty$. We define the two notions of index of $E$ (see $[4,3.6]$ or $[18,1.1 .5])$ :

1. The index of $E$ :

$$
\operatorname{Ind}_{B D H} E=\sum_{k} m_{k} m_{k}^{*} \in G_{\mathcal{Z}(M)}
$$

where $\left\{m_{k}\right\}_{k}$ is a $N$ quasi basis of the algebra $M$ and $G_{\mathcal{Z}(M)}$ is the invertible group of $\mathcal{Z}(M)$.

2. The probabilistic (or weak) index:

$$
\begin{aligned}
\operatorname{Ind} E & =\inf \{K>0: K E-I d \geq 0\} \\
& =\inf \{K>0: K\|E(a)\| \geq\|a\|, \forall 0 \leq a \in M\}
\end{aligned}
$$

Now we will study the properties of $\mathcal{P}(E)$ related with the index. In the next Proposition we prove that all the projections in $\mathcal{P}(E)$ behave like the Jones projection:

Proposition 4.2 Let $N \subseteq M$ be von Neumann algebras and $E \in E(M, N)$. Let $p \in \mathcal{P}(E)$, then

1. If we replace e by $p$, the properties 1 through 5 of Remark 3.1 are satisfied.

2. If Ind $E<\infty$ then $\|x\| \leq(\text { Ind } E)^{1 / 2}\|x p\|$ for every $x \in M$.

3. Ind $E<\infty$ if and only if $M_{1} p=M p$. 
Proof. 1) If $p=w e w^{*}$ and $w \in \mathcal{N}_{E}$, then

$$
\begin{aligned}
p x p & =w e w^{*} x w e w^{*}=w E\left(w^{*} x w\right) e w^{*}= \\
& =w w^{*} E(x) w e w^{*}=E(x) p
\end{aligned}
$$

Let $\alpha: N \rightarrow e M_{1} e$ be the isomorphism of Remark 3.1, given by $\alpha(x)=x e$ for $x \in N$. Put $p=w e w^{*}$ with $w \in \mathcal{N}_{E}$, then for $x \in N$,

$$
x p=x w e w^{*}=w\left(w^{*} x w e\right) w^{*}=A d(w) \circ \alpha \circ A d\left(w^{*}\right)(x),
$$

where $A d(w)(a)=w a w^{*}$. Note that $A d(w)\left(e M_{1} e\right)=p M_{1} p$. The other properties of $p$ are clear.

2) follows by rephrasing the proof of Prop 2.2 of [2]:

$$
\begin{aligned}
\|x p\|^{2} & =\left\|p x^{*} x p\right\|=\left\|E\left(x^{*} x\right) p\right\|=\left\|E\left(x^{*} x\right)\right\| \\
& \geq(\text { Ind } E)^{-1}\left\|x^{*} x\right\|=(\text { Ind } E)^{-1}\|x\|^{2}
\end{aligned}
$$

The last item is stated and proved in [2]

Definition 4.3 Let $N \subseteq M$ be von Neumann algebras and $E \in E(M, N)$. Denote by

$$
M^{E}=\{x \in M: E(x a)=E(a x), \forall a \in M\},
$$

the fixed algebra of the expectation $E$. Note that since $E$ is normal, $M^{E}$ is a von Neumann subalgebra of $M$.

Example 4.4 Let $N \subseteq M$ be von Neumann algebras and $E \in E(M, N)$. Suppose that $M$ is finite and $\psi$ a trace on $N$ such that $\varphi=\psi \circ E$ is also a trace. Then the expectation $E$ is "becarre" (see [9]), that is

$$
M^{E}=N^{\mathrm{c}} \quad \text { and then also } \quad M_{E}=N^{\mathrm{c}} \text {. }
$$

However, both inclusions between $M^{E}$ and $N^{\mathrm{c}}$ can be seen to be false in very easy examples.

Proof. Let $x \in M^{E}, y, z \in M, b \in N$. We need to prove that $x$ conmutes with $b$. We have

$$
\varphi((x b-b x) y)=\varphi(x b y)-\varphi(b x y) .
$$

But

$$
\varphi(b x y)=\varphi(E(b x y))=\varphi(b E(x y))=\varphi(b E(y x))=\varphi(b y x)
$$

And then, using that $\varphi$ is a trace, we obtain that $\varphi(b y x)=\varphi(x b y)$. Therefore $\varphi((x b-b x) y)=$ 0 for all $y \in M$, and $x$ belongs to $N^{\prime}$. On the other hand, if $x \in N^{\mathbf{c}}$, we have

$$
\varphi(E(x y-y x) z)=\varphi((x y-y x) E(z))=\varphi(x y E(z))-\varphi(y x E(z)) .
$$

Using that $x \in N^{\prime}$ and that $\varphi$ is a trace, we obtain that $\varphi(E(x y-y x) z)=0$ forall $z \in M$, so that $E(x y)=E(y x)$ for every $y \in M$, and then $x$ belongs to $M^{E}$. 
Lemma 4.5 Let $N \subseteq M$ be von Neumann algebras and $E \in E(M, N)$ with Ind $E<\infty$. If $p \in \mathcal{P}(E), q=u p u^{*}$ with $u \in \mathcal{N}_{E}$, and $\|p-q\|<\operatorname{Ind} E^{-1 / 2}$, then $u E\left(u^{*}\right)$ is invertible, and $q=v p v^{*}$ with $v \in \mathcal{U}_{M_{E}}$.

Proof.

$$
\begin{aligned}
\left\|1-u E\left(u^{*}\right)\right\| & \leq \operatorname{Ind} E^{1 / 2}\left\|\left(1-u E\left(u^{*}\right)\right) p\right\| \\
& =\operatorname{Ind} E^{1 / 2}\left\|p-u p u^{*} p\right\| \\
& =\operatorname{Ind} E^{1 / 2}\|(p-q) p\| \\
& \leq \operatorname{Ind} E^{1 / 2}\|p-q\|<1
\end{aligned}
$$

therefore $u E\left(u^{*}\right) \in G_{M}$ and $E\left(u^{*}\right) \in G_{N}$. Now take $v$ as the unitary part in the polar decomposition of $u E\left(u^{*}\right), v=P_{U}\left(u E\left(u^{*}\right)\right)$. It is clear by 1 of 3.4 that $v$ belongs to $N^{\mathrm{c}}$. We shall see that $v$ is also in $M^{E}$ and that it implements $q$.

By the uniqueness of the polar decomposition, we have that

$$
v=u P_{U}\left(E\left(u^{*}\right)\right)
$$

and $P_{U}\left(E\left(u^{*}\right)\right)$ lies in $N$, so that it commutes with $e$. Note that by $3.4 u$ commutes with $E\left(u^{*}\right)$ and $E\left(u^{*}\right)$ is normal, so $u$ commutes with $P_{U}\left(E\left(u^{*}\right)\right)$. Now let $x \in M$, then

$$
\begin{aligned}
E(v x) & =E\left(u P_{U}\left(E\left(u^{*}\right)\right) x\right)=P_{U}\left(E\left(u^{*}\right)\right) E(u x) \\
& =P_{U}\left(E\left(u^{*}\right)\right) u E(x u) u^{*}=v E(x u) u^{*}=E(x u) u^{*} v \\
& =E(x u) P_{U}\left(E\left(u^{*}\right)\right)=E(x v)
\end{aligned}
$$

then $v \in M^{E}$. Since $E\left(u^{*}\right)$ is invertible, $P_{U}\left(E\left(u^{*}\right)\right) \in \mathcal{U}_{N}$. Therefore $v \in \mathcal{U}_{M_{E}}$ and

$$
q=u p u^{*}=v P_{U}\left(E\left(u^{*}\right)\right)^{*} p P_{U}\left(E\left(u^{*}\right)\right) v^{*}=v p v^{*}
$$

Definition 4.6 Let $N \subseteq M$ be von Neumann algebras and $E \in E(M, N)$. From now on $\mathcal{P}(E)_{p}$ will denote the connected component of $p$ in $\mathcal{P}(E)$.

Theorem 4.7 Let $N \subseteq M$ be von Neumann algebras and $E \in E(M, N)$. Then Ind $E<\infty$ implies

$$
\mathcal{P}(E)_{p}=\mathcal{U}_{\mathcal{H}_{B}}(p):=\left\{u p u^{*}: u \in \mathcal{H}_{E}\right\}
$$

and the distance between different connected components of $\mathcal{P}(E)$ is greater than Ind $E^{-1 / 2}$.

Proof. $\mathcal{U}_{\mathcal{H}_{E}}(p)$ is connected, since $\mathcal{H}_{E}$ is connected and the map $\pi_{e}$ of 3.3 is continuous. So it is clear that $\mathcal{U}_{\mathcal{H}_{E}}(p) \subseteq \mathcal{P}(E)_{p}$.

To prove the reverse inclusion note that by Lemma $4.5, \mathcal{U}_{\mathcal{H}_{E}}(p)$ is open in $\mathcal{P}(E)$. But since the orbits of the action of $\mathcal{U}_{\mathcal{K}_{E}}$ on $\mathcal{P}(E)$ are all open and lie at distance greater than Ind $E^{-1 / 2}, \mathcal{U}_{\mathcal{H}_{E}}(p)$ is also closed in $\mathcal{P}(E)$ 


\section{1 $\mathcal{P}(E)$ as a subset of $N^{\prime} \cap M_{1}$}

Proposition 4.8 Let $N \subseteq M$ be von Neumann algebras and $E \in E(M, N)$. Suppose Ind $E<\infty$. Then $N$ is a factor if and only if e is minimal in $N^{\prime} \cap M_{1}$.

Proof. If $N$ is not a factor, let $q$ be a non trivial projection in $\mathcal{Z}(N)$. then $q e$ is a proper subprojection of $e$ in $N^{\prime} \cap M_{1}$.

Suppose now that $N$ is a factor. Let $p$ a projection in $N^{\prime} \cap M_{1}$ with $p \leq e$. Then $p e=e p=p$. Since Ind $\mathrm{E}$ is finite, by Prop. 4.2 we know that $M_{1} e=M e$. Therefore there exists $q \in M$ such that $p=p e=q e$. Then,

$$
E(q) e=e q e=e p e=p=q e,
$$

proving that $q \in N$. Moreover, $q$ is also a projection:

$$
\begin{gathered}
q^{2} e=(q e)^{2}=p^{2}=q e, \text { so that } q^{2}=q, \\
q^{*} e=(q e)^{*}=p^{*}=p=q e, \text { so that } q^{*}=q .
\end{gathered}
$$

If $b \in N, q b e=q e b=p b=b p=b q e$. Therefore $q \in \mathcal{Z}(N)$. Then, since $N$ is a factor, $q$ is a scalar, so $q=0$ or $q=1$

Proposition 4.9 Let $N \subseteq M$ be von Neumann algebras and $E \in E(M, N)$. Suppose that $N$ is a factor. Then $p q=0$ for every pair $p, q$ of projections lying in different connected components of $\mathcal{P}(E)$.

Proof. By unitary conjugation we can suppose that $q=e$. Now, assuming that $p=u e u^{*}$, with $u \in \mathcal{N}_{E}$, we have

$$
p e=u e u^{*} e={ }^{\prime} u E\left(u^{*}\right) e .
$$

If $p e \neq 0$, then $u E\left(u^{*}\right)$ is not zero, so $E\left(u^{*}\right) \neq 0$. But $E(u) E\left(u^{*}\right) \in \mathcal{Z}(N)=\mathbb{C}$, so $E\left(u^{*}\right)$ and $u E\left(u^{*}\right)$ are invertible. Proceeding as in the proof of Lemma 4.5 (but without the hipotesys of finite index), we have that $p \in \mathcal{U}_{\mathcal{H}_{E}}(e)=\mathcal{P}(E)_{\varepsilon}$

The study of $\mathcal{P}(E)$ is simpler when it is verified that $N^{\mathbf{c}} \subseteq N$ (for example for reasonable crossed products). Next theorem shows that this happens iff $\mathcal{P}(E) \subseteq \mathcal{Z}\left(N^{\prime} \cap M_{1}\right)$.

Theorem 4.10 Let $N \subseteq M$ be von Neumann algebras and $E \in E(M, N)$. Then

1. If Ind $E<\infty$ and $N^{c} \subseteq N$, then $\mathcal{P}(E) \subseteq \mathcal{Z}\left(N^{\prime} \cap M_{1}\right)$.

2. If $\mathcal{P}(E) \subseteq \mathcal{Z}\left(N^{\prime} \cap M_{1}\right)$ then $N^{c} \subseteq N$. 
Proof. First of all note that for the first implication it is enough to prove that $e \in \mathcal{Z}\left(N^{\prime} \cap M_{1}\right)$. Indeed, in that case, if $x \in N^{\prime} \cap M_{1}$ and $u \in \mathcal{N}_{E}$, then

$$
u e u^{*} x=u e\left(u^{*} x u\right) u^{*}=u\left(u^{*} x u\right) e u^{*}=x u e u^{*}
$$

since $u N^{\prime} u^{*}=N^{\prime}$. in order to prove that $e \in \mathcal{Z}\left(N^{\prime} \cap M_{1}\right)$, we will show that it equals its central carrier $C_{e}$ in that algebra. First note that $\left(N^{\prime} \cap M_{1}\right) e=\left(N^{\mathbf{c}}\right) e$. Indeed, one inclusion is clear. For the other let $x \in N^{\prime} \cap M_{1}$. As Ind $E<\infty, M_{1} e=M e$ (see [2]), so there exists $a \in M$ such that $x e=a e$. But $a$ is also in $N^{\prime}$, because if $b \in N$,

$$
a b e=a e b=x e b=b x e=b a e
$$

so that $a \in N^{\prime}$. Then we have

$$
\begin{aligned}
\left(C_{e}\right) & =\left[\left(N^{\prime} \cap M_{1}\right) e L^{2}(M, \varphi)\right] \\
& =\left[\left(N^{\mathrm{c}}\right) L^{2}(N, \psi)\right] \\
& \left.\subseteq\left[N L^{2}(N, \psi)\right]=L^{2}(N, \psi) \quad \text { (Using that } N^{\mathrm{c}} \subset N\right) \\
& =R(e)
\end{aligned}
$$

thus proving that $C_{e} \leq e$, that is $C_{e}=e$. Now suppose that $N^{c}$ is not contained in $N$, so there is an $a \in N^{\mathrm{c}}$ with $a$ not in $N$, i.e. $a-E(a)$ is not zero. If we call $\xi$ the generating and separating vector for $M$ in $L^{2}(M, \varphi)$, then the vector $(a-E(a)) \xi$ belongs to $R\left(C_{\varepsilon}\right)$ but it does not belong to $R(e)$, thus proving that e cannot be equal to its central carrier, so that it is not central

Remark 4.11 Part 1 of Theorem 4.10 and neccesity part in Proposition 4.8 are stated in $[17,1.9]$ for the $\mathrm{II}_{1}$ factor case, using strongly the existence of a trace.

\section{When is $W(E)$ finite?}

The notion of index is related with the number of elements in $W(E)$, as we will see below. It is natural to hope that $W(E)$ is finite if Ind $E<\infty$. But we shall see that the order of $W(E)$ depends both of Ind $E$ and $\operatorname{dim}(\mathcal{Z}(N))$. In particular we will also show that $W(E)$ can be infinite even if Ind $E<\infty$. We first study the case when $\operatorname{dim}(\mathcal{Z}(N))<\infty$.

Remark 5.1 Let $N \subseteq M$ be von Neumann algebras and $E: M \rightarrow N$ be a conditional expectation with Ind $E<\infty$. It is well known ([18, 1.1.2], [4, 3.19]) that

$$
\operatorname{dim}(\mathcal{Z}(M))<\infty \quad \Leftrightarrow \quad \operatorname{dim}(\mathcal{Z}(N))<\infty \quad \Leftrightarrow \quad \operatorname{dim}\left(N^{\mathrm{c}}\right)<\infty .
$$

Lemma 5.2 Let $N \subseteq M$ be von Neumann algebras and let $E: M \rightarrow N$ be a conditional expectation with Ind $E<\infty$. Then 
1. There exists $E_{1} \in E\left(M_{1}, M\right)$ such that $E_{1}(e)=\left(\operatorname{Ind}_{B D H} E\right)^{-1}$, where $\operatorname{Ind}_{B D H} E \in$ $\mathcal{Z}(M)$ and is invertible.

2. $E_{1}$ has finite index.

3. If $\operatorname{dim}(\mathcal{Z}(N))<\infty$ then $\operatorname{dim}\left(N^{\prime} \cap M_{1}\right)<\infty$.

Proof. The first two assertions are proved in $[4,3.8]$ (see also $[18,1.2]$ ). To prove 3 ) consider $E_{2}=E \circ E_{1} \in E\left(M_{1}, N\right)$. Clearly

$$
\text { Ind } E_{2} \leq \operatorname{Ind} E \text { Ind } E_{1}<\infty .
$$

Therefore Ind $E_{2}<\infty$ and $\operatorname{dim}\left(N^{\prime} \cap M_{1}\right)<\infty$

Theorem 5.3 Let $N \subseteq M$ be von Neumann algebras with $\operatorname{dim}(\mathcal{Z}(N))<\infty$ and $E: M \rightarrow N$ a conditional expectation with Ind $E<\infty$. Then $W(E)$ is finite.

Proof. Recall from Proposition 3.3 that $\mathcal{P}(E) \subseteq N^{\prime} \cap M_{1}$ which has finite dimension by Lemma 5.2. Then the unit ball of $N^{\prime} \cap M_{1}$ is compact and can not have infinitely many subsets with a fixed positive distance between eachother. Therefore $\mathcal{P}(E)$ could have just finitely many connected components (by Theorem 4.7) and $W(E)$ must be finite

\subsection{Bounds for $|W(E)|$.}

Theorem 5.4 Let $N \subseteq M$ be von Neumann algebras. Suppose that $N$ is a factor and $E: M \rightarrow N$ a conditional expectation with finite index. Then

$$
|W(E)| \leq \inf \left\{\lambda: \lambda \in \sigma\left(\operatorname{Ind}_{B D H} E\right)\right\}=\left\|\left(\operatorname{Ind}_{B D H} E\right)^{-1}\right\|^{-1} .
$$

Proof. Let $u, v \in \mathcal{N}_{E}$. Using Proposition 4.9 we have that $[u]=[v]$ in $W(E)$ if $E\left(u^{*} v\right) \neq 0$. Take different classes $\left[u_{1}\right], \ldots,\left[u_{n}\right]$ in $W(E)$ and consider $q_{i}=u_{i} e u_{i}^{*} \in \mathcal{P}(E)$ for $1 \leq i \leq n$. Therefore $E\left(u_{i}^{*} u_{j}\right)=0$ if $i \neq j$ and $\left\{q_{i}\right\}_{1 \leq i \leq n}$ is an orthogonal family of projections in $\mathcal{P}(E)$. Consider the conditional expectation $E_{1}$ of Lemma 5.2. Note that $\sum_{i=1}^{n} q_{i} \leq 1$, then

$$
1 \geq E_{1}\left(\sum_{i=1}^{n} q_{i}\right)=\sum_{i=1}^{n} E_{1}\left(q_{i}\right)=n\left(\operatorname{Ind}_{B D H} E\right)^{-1},
$$

since $\operatorname{Ind}_{B D H} E \in \mathcal{Z}(M)$ and so $E_{1}(p)=\left(\operatorname{Ind}_{B D H} E\right)^{-1}$ for $p \in \mathcal{P}(E)$. Thus $n \leq \operatorname{Ind}_{B D H} E$ (as operators) and $|W(E)| \leq\left\|\left(\operatorname{Ind}_{B D H} E\right)^{-1}\right\|^{-1}$.

Remark 5.5 Let $N \subseteq M$ be von Neumann algebras and $E: M \rightarrow N$ be a conditional expectation with Ind $E<\infty$. Then (see [7])

$$
\left\|\operatorname{Ind}_{B D H} E\right\| \leq[\text { Ind } E] \text { Ind } E \text {, }
$$

where $[x]$ denotes the entire part of $x \in \mathbb{R}$. This gives an estimation in terms of the probabilistic index for the order of $W(E)$ when $N$ is a factor. 
The main purpose of what follows is to show that, in the finite dimensional center and finite index case, sharp bounds can be found for the number of elements in $W(E)$. It will also show some of the internal structure of the inclusion $N \subseteq M$.

So let $N \subseteq M$ be von Neumann algebras. Let $E \in E(M, N)$ with Ind $E<\infty$, and $n=\operatorname{dim} \mathcal{Z}(N)<\infty$. Let $p_{1}, \ldots, p_{n}$ be the minimal central projections of $N$. Define the algebra

$$
M_{0}=\mathcal{Z}(N)^{\prime} \cap M .
$$

It is clear that $N \subseteq M_{0} \subseteq M$. Consider the conditional expectations $E_{0}: M \rightarrow M_{0}$ and $F: M_{0} \rightarrow N$ given by

$$
E_{0}(x)=\sum_{i=1}^{n} p_{i} x p_{i}, x \in M \quad \text { and } \quad F=\left.E\right|_{M_{0}} .
$$

It is clear that both $E_{0}$ and $F$ are faithful and normal.

The set $\left\{p_{1}, \ldots, p_{n}\right\}$ is stable by automorphisms of $N$, so each automorphism of $N$ induces a permutation of the indices. Then, define

$$
S: \mathcal{N}_{E} \rightarrow \mathcal{S}_{n}
$$

by $S(u)=\left.A d u\right|_{\left\{p_{1}, \ldots, p_{n}\right\}}$. That means that $S(u)=\sigma$ if $u p_{i} u^{*}=p_{\sigma(i)}$ for $0 \leq i \leq n$.

Remark 5.6 We have

$$
\operatorname{ker} S=\mathcal{N}_{E} \cap M_{0}=\mathcal{N}_{F}
$$

and

$$
\left|\mathcal{N}_{E} / \operatorname{ker} S\right| \leq n !
$$

It is also easy to see that $\mathcal{H}_{E}=\mathcal{H}_{F}$, then

$$
\operatorname{ker} S / \mathcal{H}_{E}=\mathcal{N}_{F} / \mathcal{H}_{E} \simeq W(F) \text {. }
$$

Note that both $\mathcal{N}_{F} \subseteq \mathcal{N}_{E}$ and $W(F) \subseteq W(E)$ are normal subgroups. By elementary group theory,

Therefore,

$$
\mathcal{N}_{E} / \mathcal{N}_{F} \simeq \frac{\mathcal{N}_{E} / \mathcal{H}_{E}}{\mathcal{N}_{F} / \mathcal{H}_{E}} \simeq W(E) / W(F)
$$

$$
|W(E)| \leq n !\left|\operatorname{ker} S / \mathcal{H}_{E}\right|=n !|W(F)|
$$

Theorem 5.7 Let $N \subseteq M$ be von Neumann algebras, $n=\operatorname{dim} \mathcal{Z}(N)<\infty$, and $E \in$ $E(M, N)$ with Ind $E<\infty$. Let $p_{1}, \ldots, p_{n}$ be the minimal projections of $\mathcal{Z}(N)$. Let $M_{0}=$ $\mathcal{Z}(N)^{\prime} \cap M$. Then

$$
|W(E)| \leq n !\left|W\left(\left.E\right|_{M_{0}}\right)\right| \leq n ! \Pi_{i=1}^{n}\left\|\left(\operatorname{Ind}_{B D H} E_{i}\right)^{-1}\right\|^{-1} \leq n ! K^{n},
$$

where $E_{i}=\left.E\right|_{p_{i} M p_{i}}$ and $K=\sup _{1 \leq i \leq n}\left\|\left(\operatorname{Ind}_{B D H} E_{i}\right)^{-1}\right\|^{-1}$. This inequality is sharp.

Proof. The inequality follows easily from Remark 5.6 and Proposition 2.8 (note that $\mathcal{Z}(N) \subseteq$ $\mathcal{Z}\left(M_{0}\right)$ and 2.8 applies). We will show that the equality can be realized in example 6.2 . 


\section{Examples}

In this section we mention some examples of inclusions of von Neumann algebras in which it is not difficult to calculate the normalizer $\mathcal{N}_{E}$ and the group $W(E)$.

\subsection{Projecting to the diagonal}

Let $\mathcal{R}$ be a factor, and consider the inclusion

$$
N=\mathcal{R}^{n} \subseteq \mathcal{R}^{n \times n}=M,
$$

where the conditional expectation $E$ acts by "compression to the diagonal". Then $W(E)$ is the group $\mathcal{S}_{n}$ of permutations of $n$ elements, so it has $n$ ! elements. Indeed, the map $S: W(E) \rightarrow \mathcal{S}_{n}$ of equation (8) becomes clearly a group isomorphism.

\subsection{MaXimal order for $W(E)$}

Let $B$ be a factor and $G_{0}$ a finite group of outer automorphisms of $B$. Let $E_{0}: B \times_{\alpha} G_{0} \rightarrow B$ be the canonical expectation and let $n \in \mathbb{N}$. Consider the inclusion

$$
B^{n} \subseteq\left(B \times_{\alpha} G_{0}\right)^{n \times n}
$$

with the expectation $E$ given by $E\left(\left(a_{i, j}\right)_{i, j}\right)=\Theta_{i} E_{0}\left(a_{i i}\right)$. Then

$$
|W(E)|=n !\left|G_{0}\right|^{n} .
$$

Indeed, in this example the map $S$ of equation (8) is surjective, because one can achieve every permutation of the set of minimal diagonal projections (that is, minimal projections of the center of $B^{n}$ ) by matrices with scalar entries, which belongs to $\mathcal{N}_{E}$. Using example 6.5 below, we know that Ind $E_{i}=\left|W\left(E_{i}\right)\right|=\left|G_{0}\right|$, because every inclusion $p_{i} B \subseteq_{E_{i}} p_{i}\left(B \times{ }_{\alpha} G_{0}\right)^{n \times n} p_{i}$ is a factor inclusion isomorphic to $B \subseteq E_{0} B \times_{\alpha} G_{0}$. The equality follows by using 5.6 and 2.8 .

\subsection{AN INFINITE CASE}

Here we show that, even if Ind $E<\infty$, the group $W(E)$ can be infinite. Let $A$ be an abelian infinite dimensional von Neumann algebra and consider the inclusion $N=A \oplus A \subseteq M=A^{2 \times 2}$ as diagonal matrices, with the expectation

$$
E\left(\left(\begin{array}{ll}
a_{11} & a_{12} \\
a_{21} & a_{22}
\end{array}\right)\right)=\left(\begin{array}{cc}
a_{11} & 0 \\
0 & a_{22}
\end{array}\right) .
$$

Let $p$ be a projection in $A$. Consider the matrix

$$
u_{p}=\left(\begin{array}{cc}
p & 1-p \\
1-p & p
\end{array}\right) .
$$


It is easy to see that $u_{p} \Xi N_{E}$. Since $N^{\prime} \cap M=N$ we know by 3.3 .3 that $\mathcal{H}_{E}=\mathcal{U}_{N}$. Straightforward computations show that if $p$ and $q$ are projections in $A$, then $u_{p} u_{q} \in \mathcal{U}_{N}$ iff $p=q$. Therefore the classes of $p$ and $q$ agree in $W(E)$ iff $p=q$. Since $A$ is infinite dimensional, we deduce that $W(E)$ is infinite.

Note that this example can be regarded as a cross product inclusion of algebras. Indeed, the $Z_{2}$ action on $N$ given by the permutation of the two coordinates produces a cross product algebra isomorphic to $M$. The unitary of $M$ which implements the permutation automorphism is the matrix $\left(\begin{array}{ll}0 & 1 \\ 1 & 0\end{array}\right)$.

\subsection{The Trace}

Let $N$ be a factor and consider now the inclusion

$$
N \subseteq M=N^{n \times n}
$$

with the conditional expectation being the "normalized operator valued trace". Then $W(E)$ consists of a single element.

In order to prove this fact, note that $N^{\mathrm{c}}$ consists of the matrices with entries in $\mathbb{C}$. Moreover, since the entries of matrices of $M$ and $N^{\mathbf{c}}$ commute and $E$ is trace like, we deduce that $M_{E}=N^{\mathbf{c}}$.

On the other hand $N$ is a factor and by Proposition 4.9 we have that if $u \in \mathcal{N}_{E}$ and $[u] \neq 1$ in $W(E)$ then $E(u)=0$. Let $A$ be a unitary scalar matrix in $N^{\mathbf{c}}=M_{E}$. If $E(u A)$ were not zero, it should be $[u A]=1$ and so $[u]=1$

In other words, if $u \in \mathcal{N}_{E}$ and $[u] \neq 1$, then $E(u A)=0$ for every unitary scalar matrix $A$. This means that

$$
\sum_{i=1}^{n}\left(\sum_{k=1}^{n} u_{i k} A_{k i}\right)=0
$$

Choosing the matrix $A$ to be the identity and the identity with the last sign changed, it appears that $u_{n n}=0$. Changing the other signs we can show that all the diagonal must be null. But we can also choose for $A$ the permutation matrices, so every entry of $u$ can be shifted to belong to the diagonal and so $u$ must be zero, a contradiction. Therefore $W(E)$ is the trivial group. described as

We proved that $\mathcal{N}_{E}=\mathcal{H}_{E}$ and $M_{E}=N^{\mathrm{c}}$. Therefore the normalizer of $E$ can be

$$
\mathcal{N}_{E}=\mathcal{H}_{E}=\left\{\left(n . a_{i j}\right)_{i j}: n \in \mathcal{U}_{N} \text { and }\left(a_{i j}\right) \in \mathbb{C}^{n \times n} \text { is unitary }\right\}
$$

\subsection{Discrete Crossed Products}

This example is mostly the one that justifies the introduction of the group $W(E)$ (see [6] and [14]). Take a von Neumann algebra $N$ and a discrete group $G$ of outer (and free) automorphisms of $N$, and consider $M=N \times{ }_{\alpha} G$, with the canonical conditional expectation. Then $G \subseteq W(E)$. Indeed, it is well known that $N^{c}=\mathcal{Z}(N)$. Hence $\mathcal{H}_{E}=\mathcal{U}_{N}$. Denote 
by $\lambda: G \rightarrow \mathcal{U}_{M}$ the canonical representation of $G$ in $M$. Then, since for all $g \in G, A d_{\lambda_{s}}$ commutes with $E$, we have that

$$
\left\{\lambda_{g}\right\}_{g \in G} \subseteq \mathcal{N}_{E}
$$

Moreover, if $g, h \in G$ and $\lambda_{g} \lambda_{h}^{*}=\lambda_{g h^{-1}} \in \mathcal{H}_{E}=\mathcal{U}_{N}$ then $g h^{-1} \in \operatorname{Inn}(N)$ and it must be $g=h$. Therefore the map $\Phi: G \rightarrow W(E)$ given by

$$
\Phi(g)=\left[\lambda_{g}\right]=\lambda_{g} \cdot \mathcal{H}_{E}, \quad \text { for } g \in G,
$$

is an injective homomorphism.

In general $W(E)$ is bigger than $G$, also when $G$ is finite. Indeed, in the example 6.1, the algebra $M$ can be seen as a crossed product of $N$ by the action of the integers modulo $n$, but in this case $W(E) \simeq \mathcal{S}_{n}$ (see also example 6.3). Nevertheless, when $G$ is finite and $N$ is a factor, one has that $G \simeq W(E)$.

Indeed, for every $g \in G$ we denote by $\lambda_{g}$ the corresponding unitaries in $M$. It is well known (see [4], 3.7.1) that the set $\left(\lambda_{g}\right)_{g \in G}$ is a quasi basis for $M$ and

1. $\sum_{g \in G} \lambda_{g}^{*} e \lambda_{g}=1$.

2. $E\left(\lambda_{g}^{*} \lambda_{h}\right)=\delta_{g h}$ for $g, h \in G$.

3. $|G|=\sum_{g \in G} \lambda_{g} \lambda_{g}^{*}=\operatorname{Ind}_{B D H} E=\operatorname{Ind} E$.

It is also well known that $N^{c}=\mathcal{Z}(N)$ and then $W(E) \simeq \mathcal{P}(E)$. As in $6.5, \lambda_{g} \in \mathcal{N}_{E}$, $g \in G$. Then we have a set of projections $\left\{\lambda_{g} e \lambda_{g}^{*}\right\}_{g} \in \mathcal{P}(E)$, pairwise orthogonal (by 2.), with sum 1 (by 1.). By 4.8 and 4.10 , the elements of $\mathcal{P}(E)$ are minimal projections of $N^{\prime} \cap M_{1}$ and they are central. Then

$$
W(E) \simeq \mathcal{P}(E)=\left\{\lambda_{g} e \lambda_{g}^{*}\right\}_{g} \in G
$$

Therefore the map $\rho: G \rightarrow W(E)$ given by $\rho(g)=\lambda_{g} e \lambda_{g}^{*}$ defines the natural isomorphism. In this case also

$$
\text { Ind } E=|G|=|W(E)| \text {. }
$$

\subsection{InCLUSIONS OF $\mathrm{II}_{1}$ FACTORS WiTH Ind $E<4$}

Let $N \subseteq M$ be $\mathrm{II}_{1}$ factors and $E \in E(M, N)$ with Ind $E<4$. Taking into account that $N$ is a factor and therefore $N^{\mathbf{c}}=\mathbb{C} \subseteq N$ (see [8,3.6.2(c)]), we know by Remark 3.3.3 that $W(E)=\mathcal{P}(E)$. By Proposition 4.9 we also know that the elements of $\mathcal{P}(E)$ are orthogonal projections. Analysing as usual the principal graph obtained from the derived tower (see for instance $[8,4.6]$ ), we know that the center of $N_{1}^{\mathrm{c}}:=N^{\prime} \cap M_{1}$ has dimension two or three. Assume that it is two. Then, by Theorem 4.10, the center of $N_{1}^{\mathrm{c}}$ consists of elements of the form

$$
\lambda e+\mu(1-e),
$$

with $\lambda, \mu \in \mathbb{C}$ and $e$ the Jones projection. So, if $\mathcal{P}(E)$ were not trivial, there must exist $u \in \mathcal{N}_{E}$ with $1-e=u e u^{*}$, so

$$
e+u e u^{*}=1
$$


Using the conditional expectation $E_{M}: M_{1} \rightarrow M$ given by the basic construction, we have that

$$
1=E_{M}(1)=E_{M}\left(e+u e u^{*}\right)=2\left(\operatorname{Ind}_{B D H} E\right)^{-1},
$$

so that

$$
\text { Ind } E=\operatorname{Ind}_{B D H} E=2 .
$$

By the preceeding discussion, we are allowed to consider three cases:

1. Ind $E<4$ and principal graph of type neither $\mathbf{A}_{3}$ nor $\mathbf{D}_{4}$ : This is the case considered in the discussion before, so that we have proved that $W(E)$ is trivial.

2. Ind $E=2$ (principal graph of type $\mathbf{A}_{3}$ ): It is well known in this case, by Goldman's theorem, that

$$
M=N \times_{\alpha} \mathbb{Z}_{2}
$$

so by Example 6.5 it is $W(E)=\mathbb{Z}_{2}$.

3. Ind $E=3$ with principal graph of type $\mathbf{D}_{4}$ : In this case $\mathcal{Z}\left(N^{\prime} \cap M_{1}\right)$ has three orthogonal projections, and by Example 6.5, this the situation when

$$
M=N \times_{\alpha} \mathbb{Z}_{3}
$$

so that

$$
W(E)=\mathbb{Z}_{3}
$$

\section{References}

[1] E. Andruchow, A. R. Larotonda, L. Recht and D. Stojanoff; Infinite dimensional homogeneous reductive spaces and finite index conditional expectations, Illinois J. of Math 41-1 (1997), 54-76.

[2] E. Andruchow, D. Stojanoff, "Geometry of conditional expectations and finite index", Int. Journal of Math. 5 (1994), 169-178.

[3] E. Andruchow, D. Stojanoff, "Conditional expectations with finite weak index", preprint (1995).

[4] J. Baillet, I. Denizeau, J.F. Havet, "Indice d'une Esperance Conditionelle", Comp. Math 66(1988), 199-236.

[5] Combes, Delaroche, "Groupe modulaire d'une esperance conditionnelle dans une algebre de von Neumann", Bull. Soc. Math. France 13(1975), 385-426.

[6] A. Connes, "Classification of type III factors", Ann. Ec. Norm. Sup, 1973.

[7] M. Frank, E. Kirchberg, "On conditional expectations of finite index", preprint (1995), private communication.

[8] F.M. Goodman, P. de la Harpe, V.F.R. Jones, "Coxeter Graphs and Towers of Algebras", Math. Sciences Research Institute Publications 14, Springer-Verlag. 
[9] J.-F. Havet, "Esperance conditionelle minimale", J. Oper. Th. 24 (1990), 33-55.

[10] M. Izumi, "Goldman's type theorem for index 3", Publ. Res. Inst. Math. Sci. 28, No.5, 833-843 (1992).

[11] V.F.R. Jones, "Index for subfactors", Invent. Math., 72 (1983), 1-25.

[12] R. Kadison, John R. Ringrose, "Fundamentals of the Theory of Operator Algebras", II, Academic Press, New York 1986.

[13] H. Kosaki, "Extension of Jones theory on index to arbitrary factors", J. Funct. Anal. 66 (1986), 123-140.

[14] H. Kosaki, "Characterization of Crossed Product (Properly Infinite Case)", Pacific J. of Math., 137(1989) No. 1, 159-167.

[15] A. Larotonda, L. Recht, "The orbit of a conditional expectation as a reductive homogeneous space", Monteiro, Luiz (ed.), Homage to Dr. Rodolfo A. Ricabarra. Bahia Blanca: Univ. Nacional del Sur, 61-73 (1995).

[16] R. Longo, "Index of subfactors and statistics of quantum fields I and II", Comm. Math. Phys. 126 (1989), 217-247, 130 (1990), 285-309.

[17] M. Pimsner, S. Popa, "Entropy and index for subfactors", Ann. scient. Ec. Norm. Sup., 4ta serie, 19 (1986), 57-106.

[18] S. Popa, "Classification of subfactors and their endomorphisms", AMS series CBMS Vol 86 (1995).

[19] M. Takesaki, "Conditional expectations in von Neumann algebras", J. Funct. Anal. 9, 306-321 (1972).

[20] S. Watatani, "Jones Index for C* Algebras", Memoirs AMS $83 \mathrm{~N}^{\circ} 424$ (1990).

\author{
Martín Argerami \\ Dpto. de Matemática \\ Fac. Cs. Exactas - UNLP \\ CC 172 (1900) La Plata \\ Argentina \\ martin@mate.unlp.edu.ar
}

\author{
Demetrio Stojanoff \\ Instituto Argentino de Matemática \\ Saavedra 15 3er piso \\ (1085) Buenos Aires \\ Argentina \\ demetrio@mate.dm.uba.ar
}

AMS Classification Numbers: 46L10, 46L99.

Submitted: November 26, 1997

Revised: July 1, 1998 